\title{
Learning from berberine: Treating chronic diseases through multiple targets
}

\author{
YAO Jing ${ }^{1}$, KONG WeiJia ${ }^{2} \&$ JIANG JianDong ${ }^{3 *}$ \\ ${ }^{1}$ Cancer Center, Union Hospital, Tongji Medical College, Huazhong University of Science and Technology, Wuhan 430023, China; \\ ${ }^{2}$ Institute of Medicinal Biotechnology, Chinese Academy of Medical Sciences \& Peking Union Medical College, Beijing 100050, China; \\ ${ }^{3}$ Institute of Materia Medica, Chinese Academy of Medical Sciences \& Peking Union Medical College, Beijing 100050, China
}

Received June 17, 2014; accepted July 4, 2014; published online October 30, 2014

\begin{abstract}
Although advances have been made, chemotherapy for chronic, multifactorial diseases such as cancers, Alzheimer's disease, cardiovascular diseases and diabetes is far from satisfactory. Agents with different mechanisms of action are required. The botanic compound berberine (BBR) has been used as an over-the-counter antibacterial for diarrhea in China for many decades. Recent clinical studies have shown that BBR may be therapeutic in various types of chronic diseases. This review addresses BBR's molecular mechanisms of action and clinical efficacy and safety in patients with type 2 diabetes, hyperlipidemia, heart diseases, cancers and inflammation. One of the advantages of BBR is its multiple-target effects in each of these diseases. The therapeutic efficacy of BBR may reflect a synergistic regulation of these targets, resulting in a comprehensive effect against these various chronic disorders. The safety of BBR may be due to its harmonious distribution into those targets. Although the single-target concept is still the principle for drug discovery and research, this review emphasizes the concept of a multiple target strategy, which may be an important approach toward the successful treatment of multifactorial chronic diseases.
\end{abstract}

chronic multifactorial diseases, drug treatment, berberine, multiple-target

Citation: Yao J, Kong W J, Jiang J D. Learning from berberine: Treating chronic diseases through multiple targets. Sci China Life Sci, 2015, 58: 854-859, doi: $10.1007 / \mathrm{s} 11427-013-4568-\mathrm{z}$

Berberine (BBR; Figure 1) is a natural compound isolated from Chinese herbs such as Coptis chinensis and Berberis vulgaris [1]. BBR has a molecular weight (MW) of 336.37 Da and can be easily obtained from plants or through de novo synthesis [1,2]. It has anti-bacterial properties [3], and owing to its excellent safety profiles in humans, BBR has been utilized for many decades in China as an over-the- counter medicine for bacterial diarrhea. Recent studies have indicated that BBR may be effective in treating chronic, multifactorial diseases, including diabetes, hyperlipidemia, heart diseases, cancers and inflammatory diseases. In addition, laboratory studies have identified several molecules and signaling pathways that account for its therapeu-

*Corresponding author (email: jiang.jdong@163.com) tic effects.

This review summarizes recent studies showing the clinical effects of BBR in diseases other than bacterially-caused diarrhea. Importantly, these studies indicate that the clinical effects of BBR are due to multiple molecules and/or mechanisms. Treatment of multifactorial chronic diseases with agents that regulate multiple molecular targets may be particularly effective in the future.

\section{Diabetes}

The glucose-lowering properties of BBR were first observed in 1986 [4]. Although the mechanism has not yet been fully determined, it was found to be related to several 


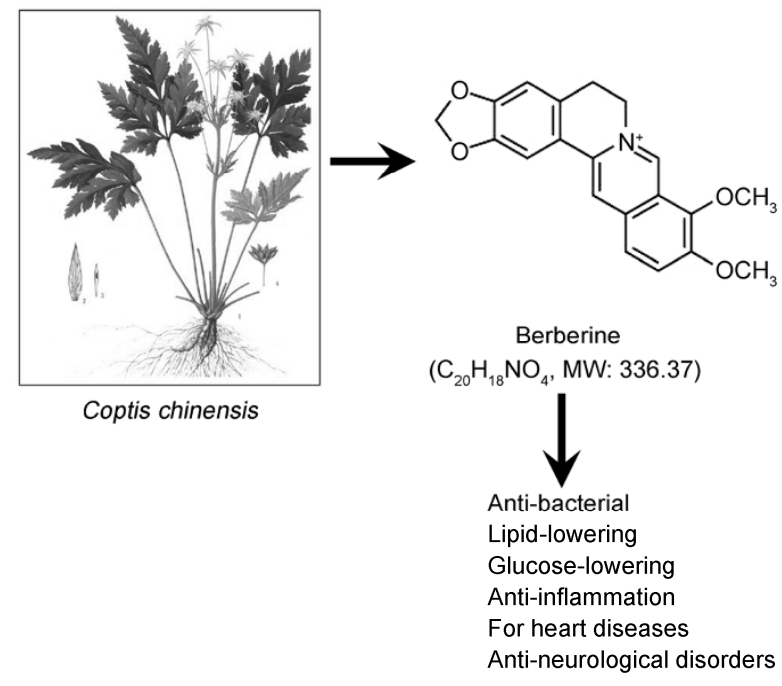

Figure 1 Chemical structure of berberine, a botanic compound with multiple effects against chronic diseases in humans.

molecular mechanisms and signaling pathways. For example, BBR could activate the AMP-activated protein kinase (AMPK) pathway through mitochondrial inhibition [5], decreasing fasting blood glucose concentration. In addition, BBR could up-regulate the level of expression of the insulin receptor (InsR) gene in liver and muscle cells, in a protein kinase D (PKD)-dependent manner, restoring their insulin sensitivity [6]. BBR was shown to inhibit PTP1B and increase the phosphorylation of InsR, insulin receptor substrate 1 (IRS1) and Akt [7]. Moreover, BBR was found to inhibit liver gluconeogenesis while promoting the differentiation of adipocytes, resulting in a net increase in glucose utilization [8]. Recent studies have suggested that gut microbiota may be involved in the mechanism by which BBR reduces blood glucose and lipid concentrations $[9,10]$.

Clinical reports have shown that BBR is safe and effective in treating type 2 diabetic patients [11]. In general, BBR alone lowered fasting blood glucose concentrations by $21 \%-36 \%$, comparable to the effects of metformin and rosiglitazone [11,13]. In addition, a clinical study in Italy suggested that BBR, when combined with standard glucose-lowering agents, such as metformin or sulfonylureas, demonstrated additive glucose-lowering efficacy in type 2 diabetic patients [12]. The main advantage of this botanic compound is its safety profile when compared with chemically synthesized glucose-lowering drugs such as rosiglitazone and metformin. For example, although the latter is not suitable for patients with chronic hepatitis and type 2 diabetes because it could lead to further deterioration in liver function, BBR was safe and effective, improving liver function in these patients while lowering blood glucose concentration [13]. In addition, animal experiments showed that BBR may have benefits in treating diabetic complications, such as endothelial dysfunction, diabetic nephropathy, and diabetic neuropathy [14].

\section{Hyperlipidemia}

BBR was first shown in 2004 to have lipid-lowering properties in animals as well as in hyperlipidemic patients [1]. Mechanistic studies have shown that BBR activates the extracellular-signal regulated kinase (ERK) pathway, stabilizing low-density-lipoprotein receptor (LDLR) mRNA and therefore increasing LDLR expression on the surface of hepatocytes [1]. This novel cholesterol-lowering mechanism differs from that of statin drugs. Detailed studies have shown that the activity of BBR is due to its effect on the responsive elements located in the LDLR mRNA 3'UTR region [1]. In addition, BBR was found to down-regulate the transcription of the gene encoding proprotein convertase subtilisin kexin 9 (PCSK9), a natural inhibitor of LDLR, suggesting another mechanism by which BBR increases LDLR expression [15]. BBR was also found to improve lipid dysregulation and prevent fatty liver by promoting the activity of AMPK [16], which has been reported to inhibit lipid synthesis [17].

Although to date no large-scale randomized double-blind clinical trials have been documented, the lipid-lowering effects of BBR have been validated by a number of independent clinical groups in and outside China. In general, BBR reduces total cholesterol concentrations by $13 \%-31 \%$, LDL cholesterol concentrations by $10 \%-25 \%$, and triglyceride concentrations by $20 \%-35 \%$ [1,11,22], with one study reporting that BBR elevates HDL cholesterol concentrations [11]. The anti-lipid mechanism of BBR differs from that of statins, which increases LDLR expression by inhibiting HMG-CoA-reductase [18]. Thus, unlike statins, BBR has no adverse effects on liver or muscle tissue. This is of particular significance for Asian populations, which are at high risk for the adverse effects of statins [19]. BBR is also useful in controlling lipid concentrations in patients with liver diseases, such as hepatitis B and C and liver cirrhosis [20], as statins increase liver enzyme concentrations in some patients [21]. Interestingly, BBR had a more pronounced effect on triglyceride concentrations than did statins [22]. The clinical advantages of BBR may result from its effects on multiple pathways of lipid and glucose metabolism [23].

\section{Heart diseases}

Laboratory research has shown that BBR possesses positive inotropic, anti- arrhythmic and vasodilator properties related to the cardiovascular system [24] by, for example, prolonging the duration of ventricular action potential [24,25]. The effects of BBR are due, at least in part, to preferential blockage of the components of the delayed rectifying potassium current, $I(\mathrm{Kr})$ and $I(\mathrm{Ks})$ [25]. BBR may also act by stimulating the $\mathrm{Na}^{+}-\mathrm{Ca}^{2+}$ exchanger [24]. Furthermore, BBR 
preferentially blocks the open state of hERG channels by interacting with specific residues [24]. Its vasodilator activity may result from undetermined multi-cellular mechanisms, which may be associated with the AMPK pathway and endothelial nitric oxide synthase [26]. Taken together, these cardiovascular effects of BBR support its clinical use in patients with heart failure or arrhythmias.

A randomized clinical trial tested the effects of BBR in 156 patients with chronic congestive heart failure [27]. Of these patients, 79 were and 77 were not treated with BBR, with all patients receiving conventional therapeutic regimens, consisting of angiotensin-converting enzyme inhibitors, digoxin, diuretics, and nitrates. The BBR-treated group showed significantly greater increases in left-ventricularejection-fraction (LVEF) and exercise capacity, significant improvements on the dyspnea-fatigue index, as well as decreased rates of ventricular premature complexes (VPCs) and long-term mortality $(P<0.02)$. A second clinical trial yielded similar results [28].

Treatment of 100 arrhythmic patients with BBR (300 mg, qid) for 1-4 weeks resulted in a $>89 \%$ reduction in premature beating in 62 patients, and $>50 \%$ in the other 38 patients, indicating that BBR significantly reduced premature beating [29]. These results were later independently reproduced [30].

\section{Cancer}

BBR has been reported to have apoptotic activity in a variety of human tumor cell lines, including hepatoma, colon cancer, lung cancer, breast cancer, and leukemia cell lines [31]. BBR may act on cancer cell DNA, DNA replication enzymes (e.g., telomerase, DNA topoisomerase), matrix metalloproteinases (MMPs) in mitochondria, the $\mathrm{Bcl} 2 / \mathrm{Bax}$ ratio, $\mathrm{p53}$, and apoptosis inducing factor (AIF) [31]. Although, to our knowledge, the in vivo anticancer activities of BBR have not been documented to date, clinical studies have shown its potential in supporting radiation therapy for cancer. For example, a randomized clinical trial involving 78 cancer patients receiving abdominal or whole pelvic radiotherapy showed that, compared with patients who did not receive BBR, those pretreated or treated with BBR (300 mg kg-1, tid) experienced significant decreases in the incidence and severity of radiation-induced acute intestinal symptoms (RIAIS; $P<0.05$ ) [32]. This suggests that BBR has a supporting effect in patients on radiation therapy. In another randomized double-blind trial [33], 90 patients with non-small cell lung cancer (NSCLC) were randomized $1: 1$ to radiation therapy plus BBR or radiation therapy plus placebo for six weeks. The rates of radiation-induced lung injury (RILI) six weeks and six months later were significantly reduced in the BBR group, whereas pulmonary function was significantly improved [33]. Despite these effects, the molecular mechanism of action of
BBR remains unclear.

A clinical study assessed the effects in 21 breast cancer patients of cholesterol-lowering natural products on secondary dyslipidemia, which frequently persists after life style changes in patients on hormone-therapy following breast cancer (HT-BC) [34]. BBR plus Red Yeast Rice (AP), combined with diet, reduced total cholesterol, LDL and triglyceride concentrations and was well tolerated by these patients.

\section{Inflammatory diseases}

The anti-inflammatory effects of BBR have been observed in a variety of human and animal tissues, including the liver [35], adipose tissue [36], vascular endothelial cells [37], and intestine [38]. Although the detailed mechanisms remain to be determined, BBR was shown to reduce the levels of expression of genes encoding pro-inflammatory cytokines, including tumor necrosis factor- $\alpha$ (TNF- $\alpha)$, interleukins (ILs), prostaglandins (PGs), cyclooxygenase-2 (COX2 ), and inducible nitric oxide synthase (iNOS), in an AMPactivated protein kinase (AMPK)-dependent manner [39]. Moreover, the anti-inflammatory effects of BBR involved inhibition of the nuclear factor-kappa $B(N F-\kappa B)$ pathway [40]. For example, in cultured cells, BBR suppressed lipopolysaccharide (LPS)-induced NF- $\mathrm{KB}$ activation and subsequent inflammation [40].

As inflammation is a key factor in the pathophysiological process of metabolic disorders, cardiovascular diseases and cancers [41], the anti-inflammatory activity of BBR may result in beneficial effects against these disorders. The anti-inflammatory effects of BBR in treating diabetes have been assessed in relative depth. BBR was found to block the development of type 1 diabetes in NOD mice through its anti-inflammatory and immune regulatory properties [42]. In addition, BBR inhibited the expression of proinflammatory cytokines in adipose tissues of $\mathrm{db} / \mathrm{db}$ diabetic and obese mice [39]. A clinical report analyzed the antiinflammatory effects of BBR in Chinese patients with type 2 diabetes [43], finding that BBR treatment for three months significantly reduced serum concentrations of proinflammatory cytokines, such as TNF- $\alpha$ and IL-6, as well as reducing blood glucose concentrations and restoring insulin sensitivity [43]. The anti-inflammatory effects of BBR have also been observed in patients with coronary heart disease. BBR treatment for $30 \mathrm{~d}$, following standard intervention, reduced serum inflammatory markers, as well as lipid concentrations, in patients with acute coronary syndrome (ACS) [44].

These anti-inflammatory properties of BBR account, at least in part, for its pharmacological efficacy against diseases such as diabetes and cardiovascular disorders. However, its molecular mechanisms, the cellular signaling pathways involved and the clinical significance of this antiinflammatory activity of BBR require further study. 


\section{Other conditions}

BBR also has effects on the nervous system. In animal models, for example, BBR treatment was shown to improve brain function (memory and learning capacity) and to relieve depression [45-47]. As these activities have not yet been verified in humans, they are not further discussed in this review.

\section{Perspectives}

Single-target therapy has been the mainstay of molecule-based drug discovery, with the goal of reducing undesired side-effects of administered agents. This strategy has achieved success in the treatment of viral infections, such as $\mathrm{HIV}-1, \mathrm{HBV}$ and HCV, using reverse transcriptase, protease, and integrase inhibitors. Drugs highly selective for single targets have also shown clinical benefits in treating non-infectious chronic diseases; however, their therapeutic efficacy is often transient, eventually failing because patients develop drug-resistance and/or side-effects.

A recent example is the withdrawal (and limited use) of thiazolidinediones (TZDs), a class of selective agonists of peroxisome proliferator-activated receptor gamma (PPAR $\gamma$ ) with glucose-lowering effects in patients with type 2 diabetes [48]. Side-effects of TZDs were observed in the cardiovascular, hepatic, and urinary systems [49-51]. The potency and selectivity of the TZD drugs toward PPAR $\gamma$ correlate positively with their side-effects [51]. The phenomenon was also seen with the cyclooxygenase-2 (Cox2) inhibitors, rofecoxib and valdecoxib [52-54].

In contrast, BBR has various therapeutic effects, with multiple molecular mechanisms of action. For example, BBR has several activities in patients with energy-related metabolic disorders, such as hyperlipidemia and type 2 diabetes, acting through several pathways. BBR up-regulates LDLR by activating ERK and inhibiting PCSK9, reduces lipid synthesis and increases glucose consumption by activating $A M P K$, up-regulates InsR by activating PKD, and decreases insulin resistance by inhibiting PTP1B and mTOR. These molecules may be components of an intact network that regulates cellular energy metabolism. The effectiveness of BBR on metabolic disorders in patients has been widely documented by independent clinical groups, both inside and outside China; this therapeutic efficacy represents the synergistic effectiveness of these molecules or pathways regulated by BBR.

Two hypotheses may explain the multiple-target nature of BBR. First, as BBR is a small-molecular-weight compound, it may be a ligand that docks into pockets of a number of proteins. Second, BBR can bind to nucleic acids, such as dsRNA. As miRNA molecules play a key role in controlling the expression of different genes, BBR in its planar form [57] might bind to grooves on the 3D structure of RNA [55,56].

BBR has shown good safety results in human applications $[1,11]$. Despite having significant clinical efficacy, the potency of its biological activity against each target is considered moderate. A balanced distribution of the chemical energy of BBR into those targets may account for its safety, making "Yin and Yang" balanced in the body.

Drugs with multiple-targets, such as aspirin, steroids and metformin, are often used to successfully manage illnesses. Therefore, although the specific- or selective-target approach still dominates, a multiple-targeted strategy might be a promising avenue in drug discovery, particularly for chronic diseases associated with multiple factors.

This review was partially supported by the National Mega-Project for Drug Research \& Development, China (to Jiang JianDong).

1 Kong W, Wei J, Abidi P, Lin M, Inaba S, Li C, Wang Y, Wang Z, Si S, Pan H, Wang S, Wu J, Wang Y, Li Z, Liu J, Jiang J D. Berberine is a novel cholesterol-lowering drug working through a unique mechanism distinct from statins. Nat Med, 2004, 10: 1344-1351

2 Huang Z J, Zeng Y, Lan P, Sun P H, Chen W M. Advances in structural modifications and biological activities of berberine: An active compound in traditional Chinese medicine. Mini Rev Med Chem, 2011, 11: 1122-1129

3 Xie Q, Johnson B R, Wenckus C S, Fayad M I, Wu C D. Efficacy of berberine, an antimicrobial plant alkaloid, as an endodontic irrigant against a mixed-culture biofilm in an in vitro tooth model. J Endod, 2012, 38: 1114-1117

4 Chen Q M, Xie M Z. Studies on the hypoglycemic effect of Coptis chinensis and berberine (in Chinese). Yao Xue Xue Bao, 1986, 21: 401-406

5 Lee Y S, Kim W S, Kim K H, Yoon M J, Cho H J, Shen Y, Ye J M, Lee C H, Oh W K, Kim C T, Hohnen-Behrens C, Gosby A, Kraegen E W, James D E, Kim J B. Berberine, a natural plant product, activates AMP-activated protein kinase with beneficial metabolic effects in diabetic and insulin-resistant states. Diabetes, 2006, 55: 22562264

6 Zhang H, Kong W J, Shan Y Q, Song D Q, Li Y, Wang Y M, You X F, Jiang J D. Protein kinase D activation stimulates the transcription of the insulin receptor gene. Mol Cell Endocrinol, 2010, 330: 25-32

7 Chen C, Zhang Y, Huang C. Berberine inhibits PTP1B activity and mimics insulin action. Biochem Biophys Res Commun, 2010, 397: 543-547

8 Xia X, Yan J, Shen Y, Tang K, Yin J, Zhang Y, Yang D, Liang H, Ye J, Weng J. Berberine improves glucose metabolism in diabetic rats by inhibition of hepatic gluconeogenesis. PLoS ONE, 2011, 6: e16556

9 Han J, Lin H, Huang W. Modulating gut microbiota as an antidiabetic mechanism of berberine. Med Sci Monit, 2011, 17: RA164-167

10 Zhang X, Zhao Y, Zhang M, Pang X, Xu J, Kang C, Li M, Zhang C, Zhang Z, Zhang Y, Li X, Ning G, Zhao L. Structural changes of gut microbiota during berberine-mediated prevention of obesity and insulin resistance in high-fat diet-fed rats. PLoS ONE, 2012, 7: e42529

11 Dong H, Wang N, Zhao L, Lu F. Berberine in the treatment of type 2 diabetes mellitus: A systemic review and meta-analysis. Evid Based Complement Alternat Med, 2012, 2012: 591654

12 di Pierro F, Villanova N, Agostini F, Marzocchi R, Soverini V, Marchesini G. Pilot study on the additive effects of berberine and oral type 2 diabetes agents for patients with suboptimal glycemic control. Diabetes Metab Syndr Obes, 2012, 5: 213-217

13 Zhang H, Wei J, Xue R, Wu J D, Zhao W, Wang Z Z, Wang S K, 
Zhou Z X, Song D Q, Wang Y M, Pan H N, Kong W J, Jiang J D. Berberine lowers blood glucose in type 2 diabetes mellitus patients through increasing insulin receptor expression. Metabolism, 2010, 59: 285-292

14 Lan T, Shen X, Liu P, Liu W, Xu S, Xie X, Jiang Q, Li W, Huang H. Berberine ameliorates renal injury in diabetic C57BL/6 mice: Involvement of suppression of SphK-S1P signaling pathway. Arch Biochem Biophys, 2010, 502: 112-120

15 Li H, Dong B, Park S W, Lee H S, Chen W, Liu J. Hepatocyte nuclear factor 1alpha plays a critical role in PCSK9 gene transcription and regulation by the natural hypocholesterolemic compound berberine. $\mathrm{J}$ Biol Chem, 2009, 284: 28885-28895

16 Kim W S, Lee Y S, Cha S H, Jeong H W, Choe S S, Lee M R, Oh G T, Park H S, Lee K U, Lane M D, Kim J B. Berberine improves lipid dysregulation in obesity by controlling central and peripheral AMPK activity. Am J Physiol Endocrinol Metab, 2009, 296: E812-819

17 Brusq J M, Ancellin N, Grondin P, Guillard R, Martin S, Saintillan Y, Issandou M. Inhibition of lipid synthesis through activation of AMP kinase: An additional mechanism for the hypolipidemic effects of berberine. J Lipid Res, 2006, 47: 1281-1288

18 Winiarska M, Bil J, Wilczek E, Wilczynski G M, Lekka M, Engelberts P J, Mackus W J, Gorska E, Bojarski L, Stoklosa T, Nowis D, Kurzaj Z, Makowski M, Glodkowska E, Issat T, Mrowka P, Lasek W, Dabrowska-Iwanicka A, Basak G W, Wasik M, Warzocha K, Sinski M, Gaciong Z, Jakobisiak M, Parren P W, Golab J. Statins impair antitumor effects of rituximab by inducing conformational changes of CD20. PLoS Med, 2008, 5: e64

19 Antons K A, Williams C D, Baker S K, Phillips P S. Clinical perspectives of statin-induced rhabdomyolysis. Am J Med, 2006, 119: $400 \mathrm{e} 9$

20 Zhao W, Xue R, Zhou Z X, Kong W J, Jiang J D. Reduction of blood lipid by berberine in hyperlipidemic patients with chronic hepatitis or liver cirrhosis. Biomed Pharmacother, 2008, 62: 730-731

21 Beltowski J, Wójcicka G, Jamroz-Wisniewska A. Adverse effects of statins-mechanisms and consequences. Curr Drug Saf, 2009, 4: 209-228

22 Kong W J, Wei J, Zuo Z Y, Wang Y M, Song D Q, You X F, Zhao L $\mathrm{X}$, Pan H N, Jiang J D. Combination of simvastatin with berberine improves the lipid-lowering efficacy. Metabolism, 2008, 57: 10291037

23 Kong W J, Zhang H, Song D Q, Xue R, Zhao W, Wei J, Wang Y M, Shan N, Zhou Z X, Yang P, You X F, Li Z R, Si S Y, Zhao L X, Pan $\mathrm{H} \mathrm{N}$, Jiang $\mathrm{J}$ D. Berberine reduces insulin resistance through protein kinase C-dependent up-regulation of insulin receptor expression. Metabolism, 2009, 58: 109-119

24 Lau C W, Yao X Q, Chen Z Y, Ko W H, Huang Y. Cardiovascular actions of berberine. Cardiovasc Drug Rev, 2001, 19: 234-244

25 Rodriguez-Menchaca A, Ferrer-Villada T, Lara J, Fernandez D, Navarro-Polanco R A, Sanchez-Chapula J A. Block of HERG channels by berberine: Mechanisms of voltage- and state-dependence probed with site-directed mutant channels. J Cardiovasc Pharmacol, 2006, 47: 21-29

26 Wang Y, Huang Y, Lam K S, Li Y, Wong W T, Ye H, Lau C W, Vanhoutte P M, Xu A. Berberine prevents hyperglycemia-induced endothelial injury and enhances vasodilatation via adenosine monophosphate-activated protein kinase and endothelial nitric oxide synthase. Cardiovasc Res, 2009, 82: 484-492

27 Zeng X H, Zeng X J, Li Y Y. Efficacy and safety of berberine for congestive heart failure secondary to ischemic or idiopathic dilated cardiomyopathy. Am J Cardiol, 2003, 92: 173-176

28 Marin-Neto J A, Maciel B C, Secches A L, Gallo Júnior L. Cardiovascular effects of berberine in patients with severe congestive heart failure. Clin Cardiol, 1988, 11: 253-260

29 Huang W M. Treating ventricular fast arrhythmias with berberine (in Chinese). Zhong Hua Xin Xue Guan Za Zhi, 1990, 18: 156

30 Jiang C G, Kuang Y T. Therapeutic efficacy of berberine in 32 arrhythmic patients (in Chinese). Zhong Guo Zhong Xi Yi Jie He Ji Jiu Za Zhi, 1998, 5: 402
31 Tillhon M, Guamán Ortiz L M, Lombardi P, Scovassi A I. Berberine: New perspectives for old remedies. Biochem Pharmacol, 2012, 84: 1260-1267

32 Li G H, Wang D L, Hu Y D, Pu P, Li D Z, Wang W D, Zhu B, Hao P, Wang J, Xu X Q, Wan J Q, Zhou Y B, Chen Z T. Berberine inhibits acute radiation intestinal syndrome in human with abdomen radiotherapy. Med Oncol, 2010, 27: 919-925

33 Liu Y, Yu H, Zhang C, Cheng Y, Hu L, Meng X, Zhao Y. Protective effects of berberine on radiation-induced lung injury via intercellular adhesion molecular-1 and transforming growth factor-beta-1 in patients with lung cancer. Eur J Cancer, 2008, 44: 2425-2432

34 Zanardi M, Quirico E, Benvenuti C, Pezzana A. Use of a lipidlowering food supplement in patients on hormone therapy following breast cancer. Minerva Ginecol, 2012, 64: 431-435

35 Lou T, Zhang Z, Xi Z, Liu K, Li L, Liu B, Huang F. Berberine inhibits inflammatory response and ameliorates insulin resistance in hepatocytes. Inflammation, 2011, 34: 659-667

36 Choi B H, Ahn I S, Kim Y H, Park J W, Lee S Y, Hyun C K, Do M S. Berberine reduces the expression of adipogenic enzymes and inflammatory molecules of 3T3-L1 adipocyte. Exp Mol Med, 2006, 38: 599-605

37 Wu M, Wang J, Liu L T. Advance of studies on anti-atherosclerosis mechanism of berberine. Chin J Integr Med, 2010, 16: 188-192

38 Gu L, Li N, Gong J, Li Q, Zhu W, Li J. Berberine ameliorates intestinal epithelial tight-junction damage and down-regulates myosin light chain kinase pathways in a mouse model of endotoxinemia. J Infect Dis, 2011, 203: 1602-1612

39 Jeong H W, Hsu K C, Lee J W, Ham M, Huh J Y, Shin H J, Kim W S, Kim J B. Berberine suppresses proinflammatory responses through AMPK activation in macrophages. Am J Physiol Endocrinol Metab, 2009, 296: E955-964

40 Jiang Q, Liu P, Wu X, Liu W, Shen X, Lan T, Xu S, Peng J, Xie X, Huang H. Berberine attenuates lipopolysaccharide-induced extracellular matrix accumulation and inflammation in rat mesangial cells: Involvement of NF- $\kappa \mathrm{B}$ signaling pathway. Mol Cell Endocrinol, 2011, 331: 34-40

41 Pickup J C. Inflammation and activated innate immunity in the pathogenesis of type 2 diabetes. Diabetes Care, 2004, 27: 813-823

42 Cui G, Qin X, Zhang Y, Gong Z, Ge B, Zang Y Q. Berberine differentially modulates the activities of ERK, p38 MAPK, and JNK to suppress Th17 and Th1 T cell differentiation in type 1 diabetic mice. J Biol Chem, 2009, 284: 28420-28429

43 Sheng Z X, Xie D H. The pro-inflammatory cytokine levels of type 2 diabetic patients and the impact of berberine therapy. New Med, 2010, 41: $177-180$

44 Meng S, Wang L S, Huang Z Q, Zhou Q, Sun Y G, Cao J T, Li Y G, Wang C Q. Berberine ameliorates inflammation in patients with acute coronary syndrome following percutaneous coronary intervention. Clin Exp Pharmacol Physiol, 2012, 39: 406-411

45 Vuddanda P R, Chakraborty S, Singh S. Berberine: A potential phytochemical with multispectrum therapeutic activities. Expert Opin Investig Drugs, 2010, 19: 1297-1307

46 Kulkarni S K, Dhir A. Berberine: A plant alkaloid with therapeutic potential for central nervous system disorders. Phytother Res, 2010, 24: 317-324

47 Ye M Z, Fu S, Pi R B, He F. Neuropharmacological and pharmacokinetic properties of berberine: A review of recent research. J Pharm Pharmacol, 2009, 61: 831-837

48 Yki-Järvinen H. Thiazolidinediones. N Engl J Med, 2004, 351: 1106-1118

49 Tolman K G. The safety of thiazolidinediones. Expert Opin Drug Saf, 2011, 10: 419-428

50 Kung J, Henry R R. Thiazolidinedione safety. Expert Opin Drug Saf, 2012, 11: 565-579

51 Cariou B, Charbonnel B, Staels B. Thiazolidinediones and PPAR $\gamma$ agonists: Time for a reassessment. Trends Endocrinol Metab, 2012, 23: 205-215

52 Fitzgerald G A. Coxibs and cardiovascular disease. N Engl J Med, 
2004, 351: 1709-1711

53 McAdam B F, Catella-Lawson F, Mardini I A, Kapoor S, Lawson J A, FitzGerald G A. Systemic biosynthesis of prostacyclin by cyclooxygenase (COX)-2: The human pharmacology of a selective inhibitor of COX-2. Proc Natl Acad Sci USA, 1999, 96: 272-277

54 Psaty B M, Furberg C D. COX-2 inhibitors-lessons in drug safety. N Engl J Med, 2005, 352: 1133-1135

55 Kumar G S. RNA targeting by small molecules: Binding of protoberberine, benzophenanthridine and aristolochia alkaloids to various
RNA structures. J Biosci, 2012, 37: 539-552

56 Maiti M, Kumar G S. Polymorphic nucleic acid binding of bioactive isoquinoline alkaloids and their role in cancer. J Nucleic Acids, 2010, 2010: 593408

57 Li Y H, Yang P, Kong W J, Wang Y X, Hu C Q, Zuo Z Y, Wang Y M, Gao H, Gao L M, Feng Y C, Du N N, Liu Y, Song D Q, Jiang J D. Berberine analogues as a novel class of the low-density-lipoprotein receptor up-regulators: Synthesis, structure-activity relationships, and cholesterol-lowering efficacy. J Med Chem, 2009, 52: 492-501

Open Access This article is distributed under the terms of the Creative Commons Attribution License which permits any use, distribution, and reproduction in any medium, provided the original author(s) and source are credited. 